\title{
Hybrid techniques and neuraxial modulation for treatment of ventricular tachycardia
}
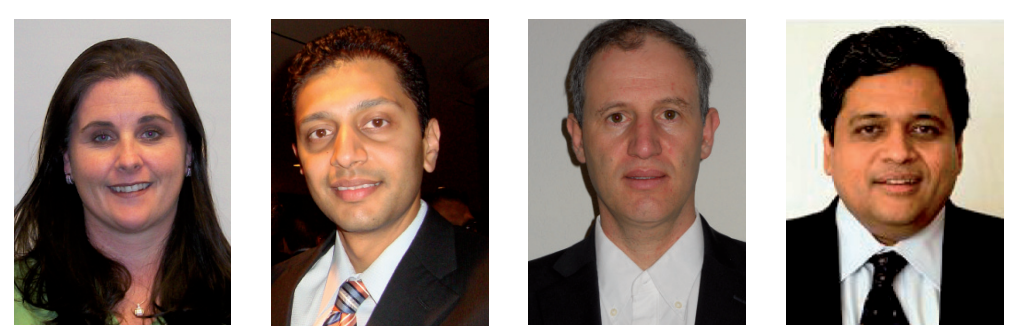

Tara Bourke', Nilesh Mathuria', Yoav Michowitz' \& Kalyanam Shivkumar ${ }^{\dagger 1}$

UCLA Cardiac Arrhythmia Center, Ronald Reagan UCLA Medical Center, David Geffen School of Medicine at UCLA, Los Angeles, CA 90095-1679, USA

†Author for correspondence: Tel. : +1 3102066433 n. Fax: +1 3107946492 n. kshivkumar@mednet.ucla.edu

Ventricular tachycardia (VT) contributes to significant morbidity and mortality for patients with structural heart disease. The implantable cardioverter-defibrillator (ICD) has been demonstrated to help in reducing mortality in this patient population [1-3]. The ICD, however, cannot prevent future shocks and also contributes to significant anxiety for patients. Anti-arrhythmic drugs also have limited efficacy and are frequently not tolerated owing to various side effects.

Catheter ablation of VT has now been shown to reduce recurrent VT episodes in numerous randomized trials [4,5]. In addition, recent publications have suggested that an early ablation procedure prior to VT development may improve patient outcomes [6]. Although percutaneous epicardial access has been well described and is relatively safe, patients with prior surgery frequently have pericardial adhesions which make a percutaneous approach difficult and/or not feasible [7]. In these patients, a hybrid surgical approach may be warranted for successful ablation of VT. However, the surgical approach requires detailed planning, coordination with cardiac surgeons and an integrated multidisciplinary approach.

Despite the advancements in substrate modification with catheter ablation, the interplay of the autonomic nervous system and development of VT is a growing avenue of research. Recent publications suggest modulation of this system may terminate and prevent future VT events. In this article, we will describe our approach in using these hybrid procedures and neuraxial modulation in the treatment of VT.

\section{Hybrid surgical approach}

In $15-30 \%$ of VTs and less often in supraventricular arrhythmias, a critical part of the reentrant circuit or the origin of the tachycardia is located epicardially [8-10]. In order to successfully ablate these arrhythmias, epicardial access must be obtained.

'6...recent publications have

suggested that an early ablation

procedure prior to VT development may improve patient outcomes."

The technique used for pericardial access was first described by Sosa et al. in 1996 [11]. This method used a Tuohy needle that slowly penetrates the pariental pericardium and enters the pericardial space; contrast injection helps verify proper placement. A wire is introduced through the needle and an introducer sheath is advanced over the wire. Usually through this sheath an ablation catheter can be positioned and moved freely within the epicardial space.

In patients with previous cardiac surgery (which account for approximately $10-40 \%$ of patients undergoing VT ablation) or prior pericarditis, pericardial adhesions may preclude successful pericardial access and catheter manipulation within the pericardial space. Soejima et al. were the first to describe the assessment of pericardial space by performing a subxiphoid window in the electrophysiology (EP) laboratory [12]. In their cohort, the anterior wall could not be reached in two out of six patients.

In a series published by the UCLA Cardiac Arrhythmia Center (CA, USA) we demonstrated that the subxiphoid window is useful for accessing the inferior left ventricle wall extending basolaterally. However, when the desired target for ablation is the anterior wall or the apex, an anterior thoracotomy should be used. Therefore, when planning for a surgical access for VT ablation in patient with previous cardiac

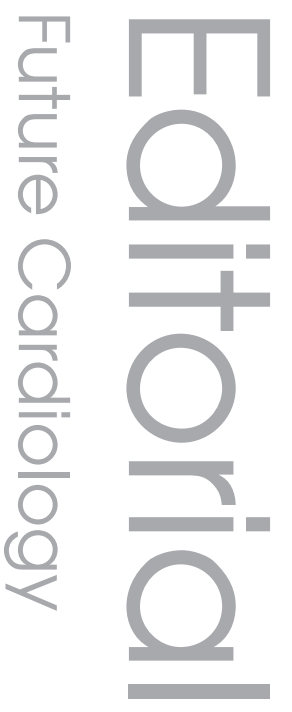

\section{Keywords}

- hybrid surgical ventricular tachycardia ablation - left cardiac sympathetic denervation $=$ thoracic epidural anesthesia

\section{future fenct $_{\text {medicine }}$ fsg}


surgery, preprocedural determination of the VT morphology and left ventricle scar location are of paramount importance in defining the preferred method of accessing the pericardium, for example by creating a subxiphoid window or anterior thoracotomy.

Several important procedural aspects should be emphasized, beginning with ECG lead placement for anterior thoracotomy which may shift owing to the incision line. Single lung ventilation is helpful for surgical exposure in anterior thoracotomy. Also with an anterior thoracotomy, a greater severity of pericardial adhesions may be observed and for extended exposure the surgeon may re-enter the thorax at a different intercostal space (see elsewhere for detailed comparison of the techniques) [13]. For procedural safety a standby perfusionist and cardiac lung machine should be available. In addition, owing to air accumulation in the pericardial space the cardiac defibrillation threshold may rise and the EP team should also be ready to cardiovert the patient internally, through his ICD, if required. No major periprocedural complications occurred in our series; however, blood loss necessitating transfusion occurred in three out of 14 patients. In conclusion the hybrid surgical approach in the EP laboratory is feasible and safe and should be considered in patients with previous cardiac surgery and a possible epicardial substrate.

\section{Thoracic epidural anesthesia}

Our standard protocol for treatment of VT includes the use of antiarrhythmic drugs, intravenous $\beta$-blockade, deep sedation and general anesthesia, as well as device reprogramming, to minimize shock therapy and endocardial and epicardial ablation procedures; however, in a minority of patients these measures fail to adequately control arrhythmias, necessitating the implementation of more novel therapeutic approaches. Sympathetic hyperactivity is an important modulator of ventricular arrhythmias, including electrical storm [14], therefore selective modulation of neural efferents to the heart is an attractive option for arrhythmia management. The antiarrhythmic effect of thoracic epidural anesthesia (TEA), including lengthening of repolarization and prolongation of refractory periods [15], has been demonstrated in animal studies [16] and its clinical utility in controlling arrhythmias in human subjects has also been described by our research team $[17,18]$.

Thoracic epidural anesthesia is a bedside procedure, performed by an anesthetist which involves placement of an epidural catheter at the T1-T2 interspace. Epidural catheter placement can be confirmed by contrast injection under fluoroscopy. A bolus of $1 \mathrm{ml}$ of $0.25 \%$ bupivicaine is followed by an infusion at $2 \mathrm{ml} / \mathrm{h}$ which results in almost immediate sympatholysis. High adrenergic state leads to ventricular arrhythmias, which in turn elevate catecholamine levels and perpetuate further arrhythmias; the effect of TEA is to break this vicious cycle. Of note thoracic epidural administration of anesthetic agents tends to have minimal effects on cardiovascular parameters including mean arterial pressure, cardiac index and central venous pressure [19].

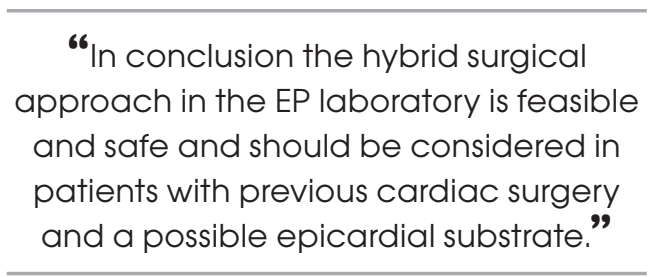

In our series, six out of eight patients had a greater than $80 \%$ reduction in arrhythmia burden following TEA. TEA may be used as a temporary measure to stabilize a patient with ongoing ischemia prior to percutaneous or surgical revascularization, prior to catheter ablation in unstable patients and may act as a bridge to cardiac surgery, cardiac transplantation or surgical left cardiac sympathetic denervation (LCSD) in patients in whom no reversible causes of VT exist or for whom no other therapeutic options remain. Benefits of TEA include ease of access and rapidity of onset (allowing any reversible precipitants to be treated). In addition TEA, by inhibiting fibers that are proximal to both the left and right stellate ganglia, could be more effective than LCSD (which is a unilateral intervention). Limitations include infection risk (which is proportional to the duration of catheter placement) and the relative contraindication in anticoagulated patients. In addition, the lack of parameters to titrate its effects (apart from absence of arrhythmias) makes accurate dosing difficult.

\section{LCSD}

For patients in whom a more permanent solution for arrhythmia control is needed, LCSD provides is a nonreversible form of neuraxial modulation. LCSD interrupts the major source of norepinephrine release in the heart. The antiarrhythmic effects of LCSD include a lengthening of ventricular refractoriness [20] and an ability to raise the ventricular fibrillation threshold [21] by approximately $70 \%$, without impairing cardiac contractility [22]. As the ventricular tachyarrhythmias of long QT 
syndrome and catacholaminergic polymorphic VT are largely triggered by sudden increases in sympathetic activity, LCSD has been predominantly used in patients with long QT syndrome and recurrent syncope despite $\beta$-blockade [23-27] and in patients with catacholaminergic polymorphic VT [27-29]. The use of LCSD in patients with arrhythmic substrates and incessant VT and VT storm has recently been described by the UCLA Cardiac Arrhythmia Center.

\section{"In our series, six out of eight patients had a greater than $80 \%$ reduction in arrhythmia burden following TEA."}

Left cardiac sympathetic denervation involves dissection and resection of the sympathetic chain from the lower part of the left stellate ganglion to the fourth thoracic ganglion. Two surgical approaches have been described, a video assisted thoracoscopic LCSD [26-30] and the traditional approach which involves a supraclavicular and retropleural approach without opening the chest $[24,31]$. Both methods have a procedure time of $45 \mathrm{~min}$. In our series, five of nine patients with refractory ventricular arrhythmias had a complete or partial reduction in arrhythmia burden following LCSD, with seven out of nine patients surviving to hospital discharge. Complications in our series included one partial Horners syndrome (which resolved after 6 months), an apical pneumothorax (which was managed conservatively) and transient facial paresthesia occurred in one patient. No procedural deaths occurred. In comparing LCSD to TEA, the immediate arrhythmia reduction may not be evident as with TEA and arrhythmias may actually increase transiently following surgery, owing to the sympathetic surge at the time of resection.

\section{Conclusion \& future perspective}

For the majority of patients, standard medical therapy in combination with endocardial and epicardial ablation may successfully treat malignant ventricular arrhythmias; however, for those patients with previous cardiac surgery in whom ventricular arrhythmias remain refractory the use of a hybrid surgical approach to ablation is an option. When all of the previous measures fail, TEA or LCSD may facilitate arrhythmia control. Despite our initial promising results using these hybrid procedures, further studies are warranted to define the clinical role of these therapeutic strategies.

\footnotetext{
Financial \& competing interests disclosure

The authors are supported by the National Heart, Lung and Blood Institute (RO1HL084261 and RO1HL067647) to Kalyanam Shivkumar. The authors have no other relevant affiliations or financial involvement with any organization or entity with a financial interest in or financial conflict with the subject matter or materials discussed in the manuscript apart from those disclosed.

No writing assistance was utilized in the production of this manuscript.
}

\section{Bibliography}

1. Buxton AE, Lee KL, Fisher JD, Josephson ME, Prystowsky EN, Hafley G: A randomized study of the prevention of sudden death in patients with coronary artery disease. Multicenter Unsustained Tachycardia Trial Investigators. N. Engl. J. Med. 341(25), 1882-1890 (1999).

2. Moss AJ, Hall WJ, Cannom DS et al:: Improved survival with an implanted defibrillator in patients with coronary disease at high risk for ventricular arrhythmia. Multicenter Automatic Defibrillator Implantation Trial Investigators. N. Engl. J. Med. 335(26), 1933-1940 (1996).

3. Moss AJ, Zareba W, Hall WJ et al.: Prophylactic implantation of a defibrillator in patients with myocardial infarction and reduced ejection fraction. N. Engl. J. Med. 346(12), 877-883 (2002).
4. Stevenson WG, Wilber DJ, Natale A et al:: Irrigated radiofrequency catheter ablation guided by electroanatomic mapping for recurrent ventricular tachycardia after myocardial infarction: the multicenter thermocool ventricular tachycardia ablation trial. Circulation 118(25), 2773-2782 (2008).

5. Tanner H, Hindricks G, Volkmer M et al:: Catheter ablation of recurrent scar-related ventricular tachycardia using electroanatomical mapping and irrigated ablation technology: results of the prospective multicenter Euro-VT-study. J. Cardiovasc. Electrophysiol. 21(1), 47-53 (2010).

6. Reddy VY, Reynolds MR, Neuzil P et al.: Prophylactic catheter ablation for the prevention of defibrillator therapy. N. Engl. J. Med. 357(26), 2657-2665 (2007).

7. Sosa E, Scanavacca M, D’Avila A, Antonio J, Ramires F: Nonsurgical transthoracic epicardial approach in patients with ventricular tachycardia and previous cardiac surgery. J. Interv. Card. Electrophysiol. 10 (3), 281-288 (2004).
8. Sacher F, Roberts-Thomson K, Maury P et al.: Epicardial ventricular tachycardia ablation a multicenter safety study. J. Am. Coll. Cardiol. 55(21), 2366-2372 (2010).

9. Aliot EM, Stevenson WG, Almendral-Garrote JM et al:: EHRA/HRS expert consensus on catheter ablation of ventricular arrhythmias: developed in a partnership with the European Heart Rhythm Association (EHRA), a registered branch of the European Society of Cardiology (ESC), and the Heart Rhythm Society (HRS); in collaboration with the American College of Cardiology (ACC) and the American Heart Association (AHA). Heart Rhythm 6(6), 886-933 (2009).

10. Kuck KH, Schaumann A, Eckardt L et al.: Catheter ablation of stable ventricular tachycardia before defibrillator implantation in patients with coronary heart disease (VTACH), a multicentre randomised controlled trial. Lancet 375(9708), 31-40 (2010). 
11. Sosa E, Scanavacca M, d'Avila A, Pilleggi F: A new technique to perform epicardial mapping in the electrophysiology laboratory. J. Cardiovasc. Electrophysiol. 7(6), 531-536 (1996).

12. Soejima K, Couper G, Cooper JM, Sapp JL, Epstein LM, Stevenson WG: Subxiphoid surgical approach for epicardial catheter-based mapping and ablation in patients with prior cardiac surgery or difficult pericardial access. Circulation 110(10), 1197-1201 (2004).

13. Michowitz Y, Mathuria N, Tung R et al.: Hybrid procedures for epicardial catheter ablation of ventricular tachycardia: value of surgical access. Heart Rhythm 7(11), 1635-1643 (2010).

14. Credner SC, Klingenheben T, Mauss O, Sticherling C, Hohnloser SH: Electrical storm in patients with transvenous implantable cardioverter-defibrillators: incidence, management and prognostic implications. J. Am. Coll. Cardiol. 32(7), 1909-1915 (1998).

15. Meissner A, Eckardt L, Kirchhof P et al.: Effects of thoracic epidural anesthesia with and without autonomic nervous system blockade on cardiac monophasic action potentials and effective refractoriness in awake dogs. Anesthesiology 95(1), 132-138; discussion 136A (2001).

16. Kamibayashi T, Hayashi Y, Mammoto T, Yamatodani A, Taenaka N, Yoshiya I: Thoracic epidural anesthesia attenuates halothane-induced myocardial sensitization to dysrhythmogenic effect of epinephrine in dogs. Anesthesiology 82 (1), 129-134 (1995).
17. Mahajan A, Moore J, Cesario DA, Shivkumar K: Use of thoracic epidural anesthesia for management of electrical storm: a case report. Heart Rhythm 2(12), 1359-1362 (2005).

18. Bourke T, Vaseghi M, Michowitz Y et al.: Neuraxial modulation for refractory ventricular arrhythmias. value of thoracic epidural anesthesia and surgical left cardiac sympathetic denervation. Circulation 121, 2255-2262 (2010).

19. Hasenbos M, Liem TH, Kerkkamp H, Gielen M: The influence of high thoracic epidural analgesia on the cardiovascular system. Acta Anaesthesiol. Belg. 39(1), 49-54 (1988).

20. Schwartz PJ, Verrier RL, Lown B: Effect of stellectomy and vagotomy on ventricular refractoriness in dogs. Circ. Res. 40(6), 536-540 (1977).

21. Schwartz PJ, Snebold NG, Brown AM: Effects of unilateral cardiac sympathetic denervation on the ventricular fibrillation threshold. Am. J. Cardiol. 37(7), 1034-1040 (1976).

22. Schwartz PJ, Stone HL: Effects of unilateral stellectomy upon cardiac performance during exercise in dogs. Circ. Res. 44(5), 637-645 (1979).

23. Schwartz PJ, Locati EH, Moss AJ, Crampton RS, Trazzi R, Ruberti U: Left cardiac sympathetic denervation in the therapy of congenital long QT syndrome. A worldwide report. Circulation 84(2), 503-511 (1991).

24. Ouriel K, Moss AJ: Long QT syndrome: an indication for cervicothoracic sympathectomy. Cardiovasc. Surg. 3(5), 475-478 (1995).
25. Schwartz PJ, Priori SG, Cerrone M et al.: Left cardiac sympathetic denervation in the management of high-risk patients affected by the long-QT syndrome. Circulation 109(15), 1826-1833 (2004).

26. Li J, Liu Y, Yang F et al.: Video-assisted thoracoscopic left cardiac sympathetic denervation: a reliable minimally invasive approach for congenital long-QT syndrome. Ann. Thorac. Surg. 86(6), 1955-1958 (2008).

27. Collura CA, Johnson JN, Moir C, Ackerman MJ: Left cardiac sympathetic denervation for the treatment of long QT syndrome and catecholaminergic polymorphic ventricular tachycardia using video-assisted thoracic surgery. Heart Rhythm 6(6), 752-759 (2009).

28. Wilde AA, Bhuiyan ZA, Crotti L et al:: Left cardiac sympathetic denervation for catecholaminergic polymorphic ventricular tachycardia. N. Engl. J. Med. 358(19), 2024-2029 (2008).

29. Atallah J, Fynn-Thompson F, Cecchin F et al:: Video-assisted thoracoscopic cardiac denervation: a potential novel therapeutic option for children with intractable ventricular arrhythmias. Ann. Thorac. Surg. 86(5), 1620-1625 (2008).

30. Li J, Wang L, Wang J: Video-assisted thoracoscopic sympathectomy for congenital long QT syndromes. Pacing Clin. Electrophysiol. 26(4 Pt 1), 870-873 (2003).

31. Schwartz PJ, Periti M, Malliani A: The long QT syndrome. Am. Heart J. 89, 378-390 (1975). 